\title{
Un guide «Gault et Millau» pour les hôpitaux?
}

Urs Metzger ${ }^{a}$,
Hermann Amstad

a Médecin-chef chirurgie, Hôpital Triemli Zurich; Président de la Commission d'accompagnement «Qualité»

b Secrétaire général de l'Académie Suisse des Sciences Médicales ASSM
Correspondance:

Dr Hermann Amstad ASSM

Petersplatz 13

CH-4051 Bâle

Tél. 0612699030

Fax 0612699039

mail@samw.ch
En septembre 2010, une association américaine de consommateurs avait publié les résultats des traitements par by-pass chirurgical de 221 centres de cardiologie (www.consumerreporthealth.org). Les données proviennent d'un registre fondé par l'American Society of Thoracic surgeons en 1989. Plus de $90 \%$ des presque 1100 centres américains de cardiologie contribuent à ce registre, dont les données sont gérées et analysées scientifiquement par le Duke Clinical Research Institute. Jusqu'alors, ce registre permettait notamment aux chirurgiens cardiologues de comparer et d'améliorer leurs propres résultats. Mais quel bénéfice le public peut-il recueillir de la publication de tels résultats? Déjà le seul fait qu'à peine 221 des 1100 centres de cardiologie aient autorisé la publication de leurs données concernant les traitements éveille des soupçons de biais de publication. Dès lors, il n'est pas étonnant que 50 des hôpitaux prêts à publier leurs résultats aient obtenu un label de qualité 3 étoiles et seulement cinq un label 1 étoile. Par ailleurs, les centres de cardiologie dont les résultats ne sont pas publiés jusqu'à ce jour, subiront une pression croissante pour les inciter à la publication. Quoi qu'il en soit - que l'on déplore cette situation ou non - les autorités responsables de ces institutions seront par là même amenées à améliorer les résultats des traitements. Et ceci est tout à fait souhaitable, à condition que la sélection des patients n'en souffre pas (voir ci-dessous).

\section{La situation en Suisse}

Le relevé et l'analyse des données concernant la qualité ont déjà une longue tradition en Suisse. L'Association pour l'étude de l'Ostéosynthèse $(\mathrm{AO})$ est parvenue, il y a 40 ans, grâce à un contrôle strict de la qualité (alors obligatoire pour les cliniques), à démontrer la supériorité de cette méthode pour le traitement des fractures et ceci, sans devoir fournir la preuve par une étude randomisée. De nombreuses sociétés de discipline médicale (infectiologie, chirurgie cardiaque, chirurgie vasculaire, orthopédie entre autres) s'efforcent aujourd'hui de relever et d'analyser la qualité des traitements sur le plan national, à l'aide de registres selon le modèle de l'American Society of Thoracic Surgeons. Il convient de saluer ces initiatives qui permettent sans aucun doute d'améliorer la qualité dans les différentes institutions. Les pouvoirs publics ont, eux aussi, entrepris de relever des indicateurs de qualité dans les établissements qu'ils subven- tionnent (par ex. Verein Outcome Zürich). Jusqu'alors, la publication de telles données a été - à juste titre très limitée. Les sources d'informations dont dispose le public ne sont ni nombreuses, ni convaincantes.

Le site Internet de Comparis présente des données - recueillies auprès des patients - concernant leur satisfaction et les taux de réadmissions, d'infections et d'erreurs dans 53 hôpitaux publics. Les résultats reposent sur une enquête téléphonique réalisée par l'institut de recherche IHA-GfK en juillet 2007 sur

\section{Mais quel bénéfice le public peut-il recueillir de la publication de tels résultats?}

mandat de Comparis auprès de 150000 personnes. Le degré de satisfaction le plus élevé a été relevé dans l'Ospedale Beata Vergine à Mendrisio avec 87 points sur 100, suivi par l'Hôpital Herisau avec 86 points et l'Hôpital cantonal d'Uri à Altdorf avec 84 points. Toutefois, cette publication ne semble pas avoir de grande influence sur le flux de patients.

En août 2010, l'Office fédéral de la santé publique (OFSP) a publié le rapport «Indicateurs de qualité des hôpitaux suisses de soins aigus 2007» (www.bag. admin/kvspi.ch). Celui-ci contient des données sur les cinq hôpitaux universitaires et 13 hôpitaux centraux resp. cantonaux qui ont consenti à la publication de leurs résultats. Les tableaux récapitulatifs des différents hôpitaux contiennent les indicateurs de qualité pour les années 2005 à 2007. Il s'agit du nombre de cas et des taux de mortalité de groupes de maladies resp. d'opérations choisis qui couvrent principalement l'assistance médicale aigüe. La cause de décès et le diagnostic resp. le traitement indiqué ne doivent toutefois pas avoir dans tous les cas un lien direct. C'est pourquoi l'OFSP stipule que «ces évaluations ne doivent pas servir de fondements pour établir des classements» et que «les résultats doivent être interprétés de façon nuancée et prudente». La publication de données concernant la mortalité lors d'opérations de hernies inguinales, de cholécystectomies laparoscopiques et d'implantations d'une prothèse totale de hanche n'a aucun sens, car dans ces interventions la mortalité ne représente pas un indicateur 
de qualité valable. Cependant, conformément à la situation juridique actuelle, l'OFSP souhaite introduire l'année prochaine l'obligation de publier ces données (pour 2008) pour tous les hôpitaux. Il convient de tenir compte du fait que l'OFSP ne dispose en Suisse d'aucun indicateur de qualité à part les taux de mortalité et le nombre de cas.

De nombreux hôpitaux ont commencé à publier, avec leurs rapports annuels, un rapport de qualité détaillé avec des indications concernant le nombre de cas et la mortalité ainsi que les résultats d'enquêtes relatives à la satisfaction des patients, des prescripteurs et des collaborateurs. De telles initiatives doivent être saluées lorsqu'elles sont intelligibles et transparentes.

\section{Situation juridique}

La garantie de la qualité des traitements médicaux est légalement ancrée dans l'article 58 de la LAMal et dans l'article 77 de l'OAMal. Ces articles exigent que les fournisseurs de prestations ou leurs organisations élaborent des concepts et des programmes en matière d'exigences de la qualité des prestations et de promotion de la qualité. Les modalités du déroulement et son financement doivent être définis dans les contrats de tarifs ou dans des contrats d'assurance qualité spécifiques avec les assureurs ou leurs associations. Depuis le 1.1.2009, un nouvel article 22a LAMal est entré en vigueur, selon lequel «les fournisseurs de

\section{Les sociétés de discipline médicale de la FMH doivent à présent élaborer pour leurs domaines respectifs des indicateurs de qualité précis, pertinents et valides}

prestations doivent communiquer aux autorités fédérales compétentes les données qui sont nécessaires pour surveiller l'application des dispositions de la présente loi relatives au caractère économique et à la qualité des prestations». Conformément à l'alinéa $\mathrm{f}$ ceci englobe explicitement les «indicateurs de qualité médicaux». Les données relevées par l'OFS sont publiées par l'Office fédéral de la santé publique (OFSP).

\section{Les indicateurs de qualité}

La qualité des structures et des procédures peut être évaluée comparativement dans le domaine de la gastronomie et dans celui des soins stationnaires (installations, propreté, amabilité du personnel, temps d'attente, etc.). En revanche, la qualité des résultats dans le domaine de la santé est largement plus complexe et est loin de n'être qu'une question de goût!

Les sociétés de discipline médicale de la FMH étaient et restent les garants d'une haute qualité de l'assistance médicale dans notre pays. Elles doivent à présent élaborer pour leurs domaines respectifs des indicateurs de qualité précis, pertinents et valides, et non plus se limiter à relever les taux de mortalité des différents hôpitaux. Ceux-ci doivent - en plus des indications de l'âge et du sexe - définir les caractéristiques des patients pour permettre ainsi une comparaison des données reflétant la qualité des résultats (composition du collectif des patients).

\section{Financement}

Un relevé sérieux de la qualité des résultats en médecine n'est pas gratuit. Il exige beaucoup de temps et d'effectifs. C'est là que la comparaison avec la gastronomie devient boiteuse! L'Association nationale pour le développement de la qualité dans les hôpitaux et les cliniques (ANQ) manque de moyens financiers. Selon Arnold Bachmann, vice-président de l'ANQ et directeur de l'Hôpital cantonal des Grisons, l'introduction d'un système national de mesure de la qualité exigerait un investissement de 20 millions de francs (NZZ 10.9.2010). Les fournisseurs de prestations doivent disposer des fonds nécessaires à l'élaboration de concepts et programmes pour le relevé, l'analyse et la publication des données concernant la qualité des traitements médicaux, tel que l'exige la loi. Le législateur s'est exprimé clairement à ce sujet, à savoir que les modalités des programmes de qualité et leur financement doivent être définis dans les contrats de tarifs ou en particulier dans les contrats d'assurance qualité avec les assureurs ou leurs associations.

«La qualité a son prix», dit-on - il en va de même pour qu'un relevé, une analyse et la publication de données concernant la qualité soient réellement fiables.

\section{Un regard vers I'avenir}

Afin d'éviter une profusion de données absurdes et ambiguës concernant la qualité, le Sénat de l'Académie Suisse des Sciences Médicales (ASSM) a publié en mai 2009 des recommandations «Relevé, analyse et publication de données concernant la qualité des traitements médicaux» dans Bulletin des médecins suisses [1]. Ces recommandations comprennent notamment une check-list contenant 18 points relatifs à la pertinence (voir page suivante), l'exactitude et la compréhension des données publiées. La FMH renvoie, elle aussi, à ces recommandations. Elle exige aussi que des indicateurs de qualité soient définis en collaboration avec le corps médical. Selon l'ASSM et la FMH, chaque publication concernant la qualité des prestations médicales devra à l'avenir remplir les critères définis, dans le sens d'une déclaration, resp. d'un label de qualité. A moyen terme, l'«Organisation nationale pour l'assurance de la qualité et la sécurité des patients» (encore à créer et déjà réclamée par une initiative parlementaire) aura pour mission d'exiger de chaque fournisseur de prestations, le respect de ces critères.

Afin d'encourager, de faciliter et d'accompagner leur mise en pratique, l'ASSM constitue une commis- 
sion d'accompagnement «Recommandations qualité». La sensibilisation et le conseil au corps médical ainsi que la vérification sporadique des publications quant à l'observation des recommandations constituent les missions de cette commission. De plus, la praticabilité des recommandations elles-mêmes devrait être vérifiée avec les responsables de la qualité.

\section{Référence}

1 ASSM. Relevé, analyse et publication de données concernant la qualité des traitements médicaux. Bull Méd Suisses. 2009;90(26/27):1044-54.

\section{Check-list}

Lors de la publication des rapports concernant la qualité, leur auteur doit mentionner les critères qui figurent sur cette liste et qui sont remplis. La transparence sur la manière dont les données ont été relevées et interprétées est ainsi possible.

\section{Pertinence}

A. Les indicateurs choisis représentent-ils la qualité des traitements médicaux?

oui/non/en partie

B. L'indicateur concerne-t-il la structure, un processus ou le résultat?

C. Le responsable du choix des indicateurs est-il mentionné? structure/processus/ résultat

2. Justesse oui/non/en partie

A. Les indicateurs sont-ils définis de manière précise?

B. La totalité des patients chez qui l'indicateur est mesuré, est-elle clairement décrite?

C. Existe-t-il des indications concrètes sur le nombre de patients chez lesquels l'indicateur a été relevé?

D. Dans le cas d'une enquête auprès des patients, existe-t-il des indications sur le taux de réponse?

E. Les instruments servant à la mesure des indicateurs sont-ils valides et fiables?

F. Des indications sur le personnel qui a recueilli les données sont-elles mentionnées dans le rapport?

G. La source des données est-elle décrite?

H. La durée de la mesure et de l'intervalle de temps sont-ils indiqués?

I. Les données nécessaires à une éventuelle rectification de la composition du collectif des patients (casemix) ont-elles été recueillies?

J. La qualité des données relevées a-t-elle été contrôlée?

oui/non/en partie oui/non/en partie

K. Y-a-t-il des indications sur la manière dont on a procédé avec les données manquantes?

L. La présentation des résultats est-elle adéquate et intelligible?

M. La réalisation de la comparaison avec d'autres fournisseurs de prestations est-elle décrite de manière détaillée?

N. Points d'ordre général
a. Qui garantit la justesse des données et des interprétations?
indiqué/non indiqué
b. A-t-on fourni des indications concernant le financement du projet?
oui/non
c. La commission d'éthique a-t-elle donné, si nécessaire, son accord? oui/non/pas nécessaire
d. Les patients ont-ils donné, si nécessaire, leur accord?
e. Qui est propriétaire des données recueillies? oui/non/pas nécessaire Les données sont-elles accessibles? oui/non

f. A-t-on réalisé un audit externe et indépendant des données recueillies?

oui/non

\section{Intelligibilité}

Les résultats sont-ils présentés de façon intelligible et interprétés de manière compréhensible? 